\title{
Colonic pericrypt sheath cells: characterisation of cell type with new monoclonal antibody
}

\author{
P I RICHMAN, R TILLY, J R JASS, * W F BODMER \\ From the Director's Laboratory, Imperial Cancer Research Fund, Lincoln's Inn Fields, London, and the \\ *Imperial Cancer Research Fund Colorectal Cancer Unit, St Mark's Hospital, and the Medical College of \\ St Bartholomew's Hospital, London
}

SUMmARY A new monoclonal antibody, PR 2D3, was raised against a crude homogenate of normal colorectal mucosa and found to react with the cells in the pericrypt sheath. It also reacted with smooth muscle throughout the body and, in specific sites, with those mesenchymal cells known as myofibroblasts. It did not react with cardiac or skeletal muscle, nor with fibroblasts. PR 2D3 is an $\mathrm{IgG}_{1}$ antibody and identifies a membrane component of about $140 \mathrm{~K}$ molecular weight.

The pericrypt cells have been described as fibroblasts, but in view of the specificity of PR 2D3 for smooth muscle, and its selective staining of the colonic pericrypt cells, this cell type was re-examined for other smooth muscle properties. Ultrastructurally, the cells had many characteristics in common with smooth muscle and were identical with the myofibroblasts of the umbilical cord. On immunocytochemical examination they were found to contain desmin, myosin, and filamin. The confirmation that the pericrypt cells are myofibroblasts suggest that they have both contractile and secretory roles.

A series of monoclonal antibodies raised against scrapings from normal human colorectal mucosa included PR 2D3, which identified the cells of the colonic pericrypt sheath and reacted with the smooth muscle of the muscularis mucosae and muscularis propria. It also stained smooth muscle throughout the body and reacted with specific populations of mesenchymal cells in other sites. Some of these-for example, in the umbilical cord ${ }^{1}$ and pulmonary alveolar septa ${ }^{2}$ - have been described as myofibroblasts. The antibody did not, however, react with cardiac or skeletal muscle, nor with fibroblasts.

The cells of the pericrypt sheath have been described as fibroblasts, ${ }^{3}$ but the selective staining of these cells by PR 2D3, and its specificity for smooth muscle and myofibroblasts, suggested that the pericrypt cells belonged to the smooth muscle or myofibroblast lineage. We therefore re-examined the pericrypt cells for other smooth muscle properties and re-evaluated their nature and function.

In this paper we present the details of the production and characterisation of the monoclonal antibody PR 2D3 and the ultrastructure of the pericrypt cells in comparison with that of the myofibroblasts of the umbilical cord. The pericrypt cells were also

Accepted for publication 30 January 1987 studied immunocytochemically, and their reactions characterised with PR 2D3 and other antibodies directed against known components of smooth muscle.

\section{Material and methods}

MONOCLONAL ANTIBODY PR 2D3

Fresh samples of normal large intestine were pinned on to a cork board and after thorough rinsing in cold sterile phosphate buffered saline A (pH 7.4) (PBS-A) the mucosa was dissected from the underlying submucosa by scraping with a scalpel. Mucosal scrapings $(0.2 \mu \mathrm{m})$ were snap frozen in liquid nitrogen and mechanically vibrated to a powder in a polypropylene phial containing a tungsten ball bearing. The powder was emulsified in $0.2 \mathrm{ml}$ Freund's Complete Adjuvant and $0.2 \mathrm{ml}$ PBS-A. Balb/c mice were immunised intraperitoneally with this material at six weeks, two weeks, and four days before fusion. The boosting immunisations were without Freund's Adjuvant. The spleen cells of one mouse were fused with the 8-azaguanine resistant $\mathrm{Balb} / \mathrm{c}$ myeloma cell line P3/NS1/1-Ag-4-1 (NS1) using 50\% polyethylene glycol 4000 (Merck) in RPMI 1640 medium. Resultant hybrid colonies were selected in RPMI 1640 supplemented with $20 \%$ fetal calf serum (FCS), $10^{-4} \mathrm{M}$ 
hypoxanthine, $1.6 \times 10^{-5} \mathrm{M}$ thymidine and $10^{-5} \mathrm{M}$ methotrexate (HAT). For screening, we used frozen sections of normal colon mounted on 10-well multiwell slides (CA Hendley-Essex Ltd). Hybridoma PR 2D3 was cloned twice by picking single cells with a drawn out Pasteur pipette. Colonies were grown up, weaned off HAT, and aliquots frozen to provide stocks for further use. Ouchterlony immunodiffusion $^{4}$ shows that PR 2D3 is an $I^{2} G_{1}$ class antibody.

\section{OTHER ANTIBODIES}

Mouse monoclonal antibody AC4 against collagen type IV was a gift from Dr J Barteck, (Imperial Cancer Research Fund). Mouse monoclonal antibodies to actin binding protein (filamin), PM6/372, and myosin, PM1/211, were kindly provided by Dr M Wilkinson, Royal College of Surgeons of England, London. Rabbit polyclonal antibody to desmin was bought from Euro-Diagnostics BV, Holland. Peroxidase conjugated rabbit antimouse, swine antirabbit, and fluorescein conjugated rabbit antimouse immunoglobulins were purchased from Dako, Copenhagen, Denmark.

\section{IMMUNOCYTOCHEMICAL METHODS}

Fresh samples of normal large intestine and colorectal tumours were obtained from the Imperial Cancer Research Fund Colorectal Cancer Unit, St Mark's Hospital, London. These were snap frozen in liquid nitrogen and stored at $-70^{\circ} \mathrm{C}$. Frozen samples of normal tissues were provided by Dr LG Bobrow, Department of Histopathology, University College Hospital Medical School, London.

Tissues were examined by indirect immunoperoxidase and immunofluorescence techniques. Cryostat tissue sections ( $6 \mu \mathrm{m}$ thick) were picked up on $0.1 \%$ poly-l-lysine coated slides. These were fixed in acetone for 10 minutes at room temperature. All monoclonal antibodies were used as unconcentrated hybridoma supernatants. The rabbit polyclonal antidesmin antibody was used at a dilution of $1 / 10$ in PBS-A. Peroxidase conjugated rabbit antimouse and swine antirabbit immunoglobulins were diluted 1/50 in PBS-A containing 5\% normal human serum. Fluorescein conjugated rabbit antimouse immunoglobulin was diluted 1/40 in PBS-A. Sections were incubated with primary and secondary antibodies for 30 minutes at room temperature. The substrate for the peroxidase reactions was a freshly prepared solution of diaminobenzidine (Sigma), $5 \mathrm{mg}$ in $10 \mathrm{ml}$ Tris hydrocholoric acid, $\mathrm{pH} 7 \cdot 6$, containing $0.03 \%$ hydrogen peroxide. The reaction was stopped after five minutes by washing in tap water. Slides were counterstained in Meyer's haematoxylin, dehydrated in a graded alcohol series, cleared in xylene and mounted in DPX (BDH). For immunofluorescence, slides were mounted in Gelvatol 20/30 (Monsanto, Springfield, Massachusetts, USA). Formalin fixed paraffin embedded sections were examined by the indirect immunoperoxidase technique, as described above.

Immunocytochemical staining was controlled by using non-hybridoma tissue culture medium (RPMI 1640 with $10 \%$ FCS) as the primary layer. In addition, for immunoperoxidase staining, the second antibody-enzyme conjugates and diaminobenzidine solution were also used individually. For immunofluorescence, the fluorescein conjugated antibody was used alone. Non-specific staining by these reagents was not observed.

\section{ELECTRON MICROSCOPY AND}

IMMUNOELECTRON MICROSCOPY

For electron microscopy, multiple $1 \mathrm{~mm}^{3}$ tissue cubes were fixed in glutaraldehyde, post-fixed in osmium tetroxide, and embedded in Araldite. Representative areas were selected from $1 \mu \mathrm{m}$ sections.

For immunoelectron microscopy, tissues were fixed in $1 \%$ monomeric glutaraldehyde in phosphate buffer (pH 7.3). After being rinsed in ammonium chloride $(0.5 \mathrm{M}$ in phosphate buffer) for two hours tissues were dehydrated in ascending alcohols to $70 \%$. They were then moved from room temperature to $-30^{\circ} \mathrm{C}$ for all further processing. Tissues were taken to $95 \%$ ethanol and soaked in a series of Lowicryl-ethanol mixtures at dilutions of $2 / 1,1 / 1$, and $1 / 2$, respectively for two hours each. They were then embedded in Lowicryl. Polymerisation of Lowicryl was at $-30^{\circ} \mathrm{C}$ using an ultraviolet (wavelength $360 \mathrm{~nm}$ ) for at least 48 hours.

Sections of the tissues embedded in Lowicryl were mounted on nickel grids and incubated in $4 \%$ normal goat serum in PBS-A for $\mathbf{3 0}$ minutes. Incubation with primary antibody (PR 2D3) was for 16 hours. After washing grids were incubated with goat antimouse immunoglobulin conjugated to colloidal gold particles $5 \mathrm{~nm}$ in diameter (Janssen Pharmaceuticals), at a dilution of 1/50 in PBS-A for one hour at room temperature. Grids were again washed in PBS-A and distilled water before being viewed in the electron microscope. Further staining was occasionally carried out in $1 \%$ osmium tetroxide for two minutes, followed by lead nitrate and uranyl acetate for one minute and 30 seconds, respectively.

\section{PROTEIN IMMUNOBLOTTING}

We adapted the protein immunoblotting technique from the method of Towbin et al. ${ }^{5}$ Five grams of freshly obtained smooth muscle dissected from rectal muscularis propria was finely diced with scalpels and stirred magnetically for 60 minutes at $4^{\circ} \mathrm{C}$ in lysis buffer $(1 \%$ Nonidet P-40, $0.5 \%$ nordeoxycholate, 


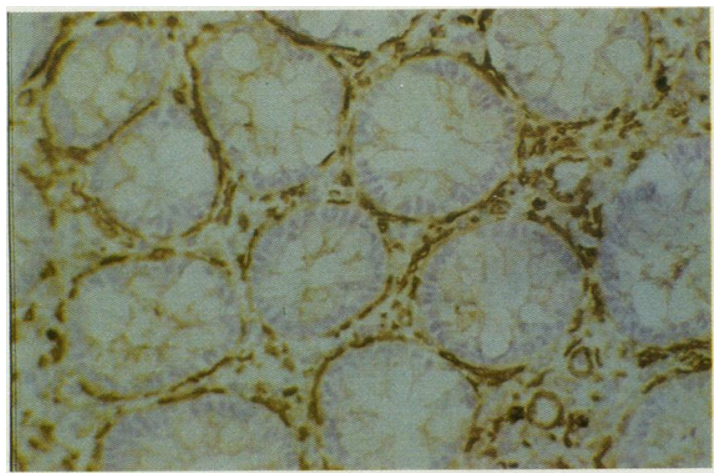

Fig 1 Colon, transverse section: pericrypt cells stained by PR 2D3. (Immunoperoxidase, cryostat section.)

$1 \mathrm{mM}$ edetic acid, $150 \mathrm{mM}$ sodium chloride, $50 \mathrm{mM}$ Tris base, $\mathrm{pH} 8 \cdot 3$ ). The supernatant was clarified by centrifugation at $1500 \mathrm{rpm}$ and separate aliquots were mixed with sample buffer ( $2 \%$ sodium dodecylsulphate (SDS), $10 \%$ glycerol, $80 \mathrm{mM}$ Tris-hydrochloric acid (pH 6.8) with or without $\beta$-mercaptoethanol. Some of the samples were boiled for five minutes before loading on to the gel. The proteins were then separated by electrophoresis in one dimension on a $7.5 \%$ (weight/volume) polyacrylamide gel overlaid with a $3 \%$ (weight/volume) polyacrylamide stacking gel. The running buffer was composed of $25 \mathrm{mM}$ Tris base, $192 \mathrm{mM}$ glycine, and $0 \cdot 1 \%$ sodium dodecyl sulphate. Prestained protein molecular weight markers (BRL) were run simultaneously. Proteins were transferred on to nitrocellulose paper overnight at $4^{\circ} \mathrm{C}$. The transfer buffer comprised $25 \mathrm{mM}$ Tris base, $195 \mathrm{mM}$ glycine, and $20 \%$ methanol. Non-specific binding of monoclonal antibodies to the nitrocellulose paper was blocked with $3 \%$ bovine serum albumin in PBS-A for 60 minutes, followed by washing in PBS-A. The nitrocellulose paper strips were incubated with unconcentrated hybridoma supernatant or with RPMI 1640 containing $10 \%$ FCS for four hours at room temperature. These were then extensively washed in PBS-A and incubated for a further 60 minutes with peroxidase conjugated rabbit antimouse IgG at a dilution of $1 / 100$ in PBS-A. After six further washes in PBS-A, and emersion in a saturated solution of 4-chloro-1-naphthol (Koch-Light Laboratories Ltd) in methanol, diluted 1/100 in PBSA, containing $0.1 \%$ hydrogen peroxidase, transferred protein could be seen.

\section{Results}

Antibody PR 2D3 did not react with paraffin embedded, formalin fixed tissue. All immunocytochemical staining results refer to acetone fixed cryostat sections.

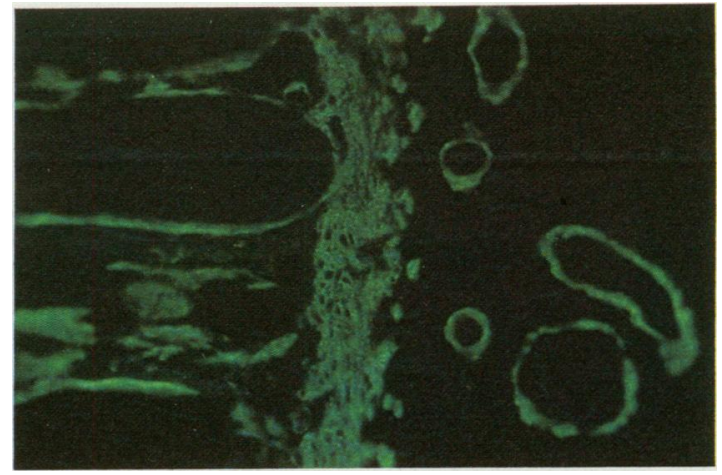

Fig 2 Colon: pericrypt cells, muscularis mucosae, and submucosal blood vessels stained by PR 2D3. (Immunofluorescence, cryostat section.)

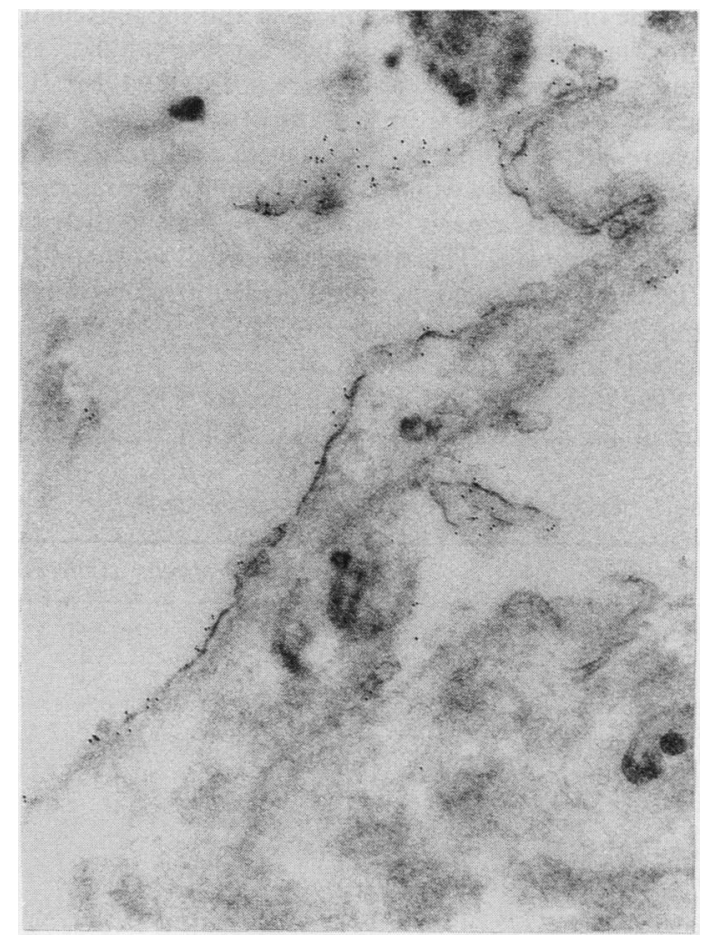

Fig 3 Electron micrograph showing immunolocalisation of $P R 2 D 3$ to smooth muscle membranes. (Immunogold.)

ANATOMICAL DISTRIBUTION OF PR 2D3

POSITIVE CELLS IN THE LARGE INTESTINE AND OTHER PARTS OF THE GASTROINTESTINAL

TRACT

Pericrypt mesenchymal cells were strongly labelled by PR 2D3. They formed a continuous sheath closely applied around the crypts of the colon and rectum, and could be seen arising from the muscularis mucosae where crypt bases abutted on this layer (figs 1 and 2). Vascular smooth muscle and the smooth 


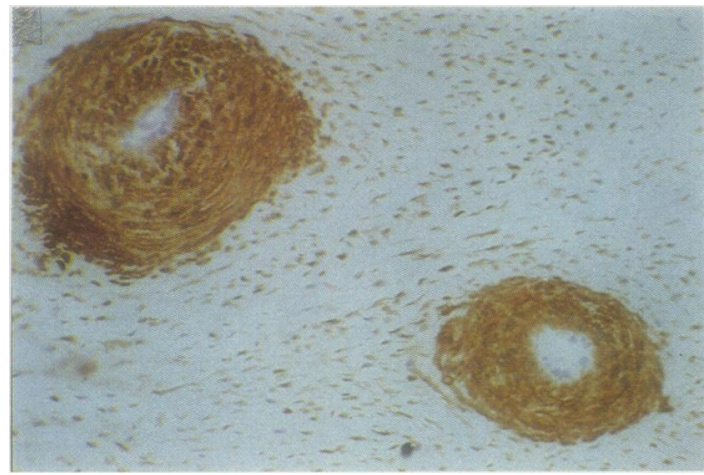

Fig 4 Umbilical cord: stellate myofibroblasts and vascular smooth muscle stained by PR 2D3. (Immunoperoxidase, Cryostat section.)

muscle of the muscularis mucosae and the muscularis propria were also stained, but nerves, ganglia, and interstitial connective tissue were not stained. PR 2D3 reactivity had a clear membrane distribution in areas where smooth muscle fibres were sectioned transversely, especially when seen by immunofluorescence. This contrasts clearly with the cytoplasmic distribution of myosin. The membrane distribution of PR 2D3 was confirmed by immunoelectronmicroscopy. Fig 3 shows the localisation of PR 2D3 to smooth muscle membranes.

The distribution of cells positive for PR 2D3 in the small intestine was similar to that seen in the colon. In

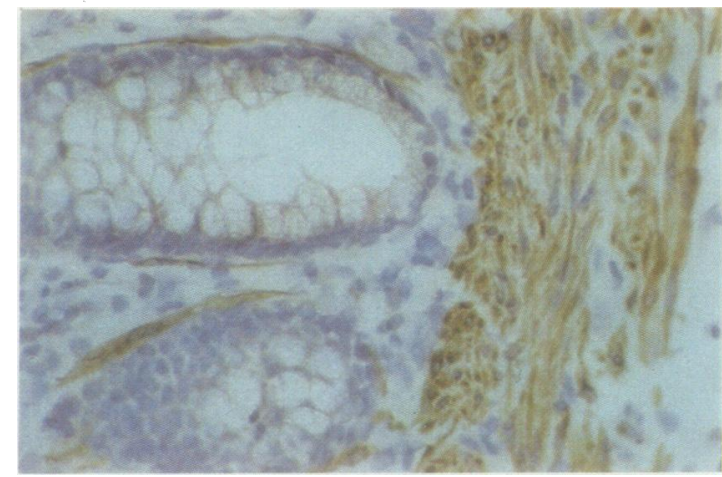

Fig 5 Colon: pericrypt cells stained for desmin.

(Immunoperoxidase, Cryostat section.)

addition to pericrypt cells, the antibody stained long straight fibres which extended upwards within the lamina propria of the villi. Positive fibres were also present around gastric glands where they formed a continuous mesenchymal sheath. In the oesophagus only vascular smooth muscle reacted with PR 2D3.

ANATOMICAL DISTRIBUTION OF CELLS POSITIVE TO PR 2D IN OTHER NORMAL TISSUES

The table summarises the distribution of cells reacting to PR 2D3. The antibody stained vascular and other smooth muscle in all tissues examined. It also reacted with mesenchymal cells in specific sites, some of which

Table Reactivity of $\operatorname{Pr} 2 D 3$ with normal human tissues

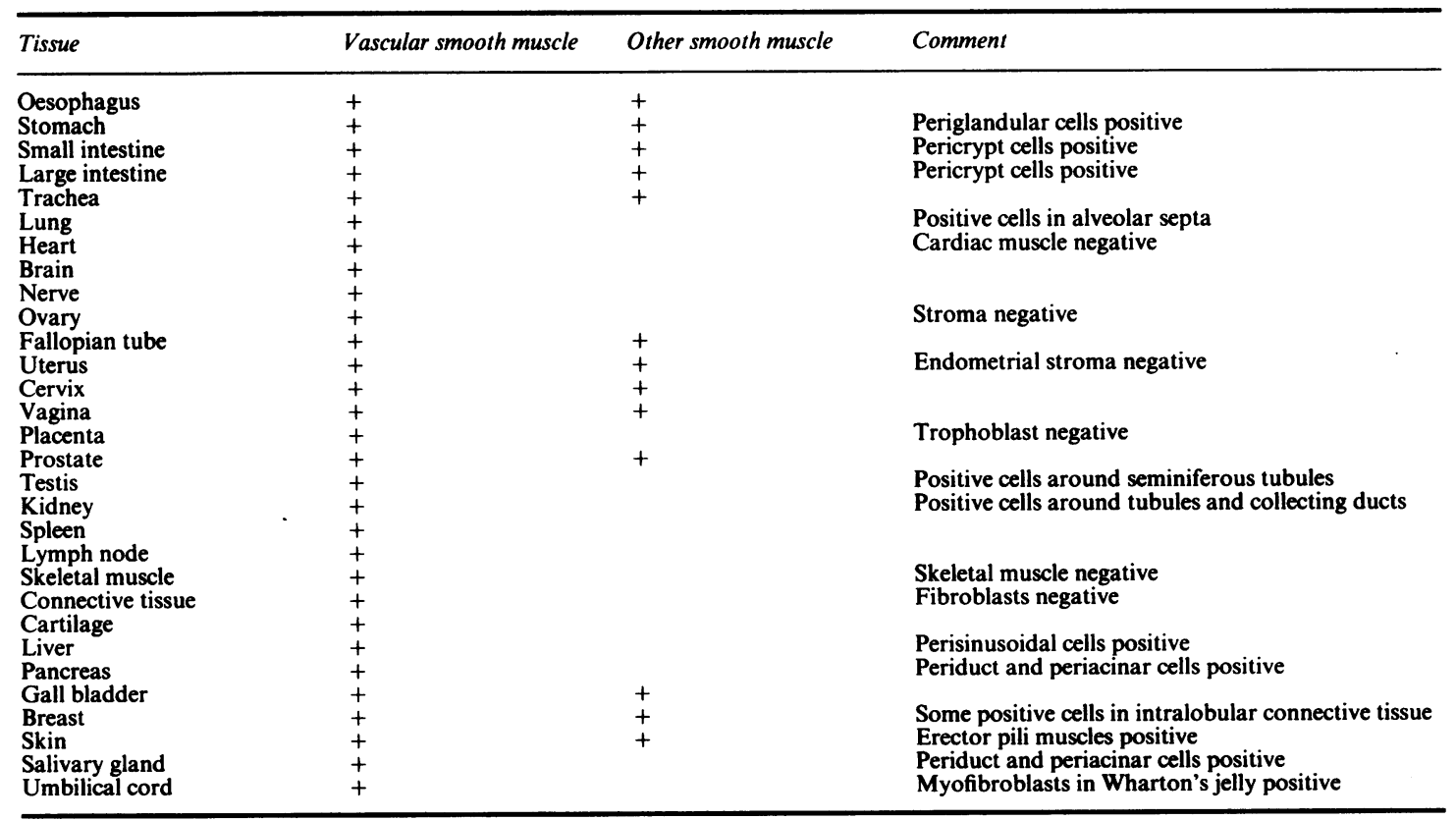




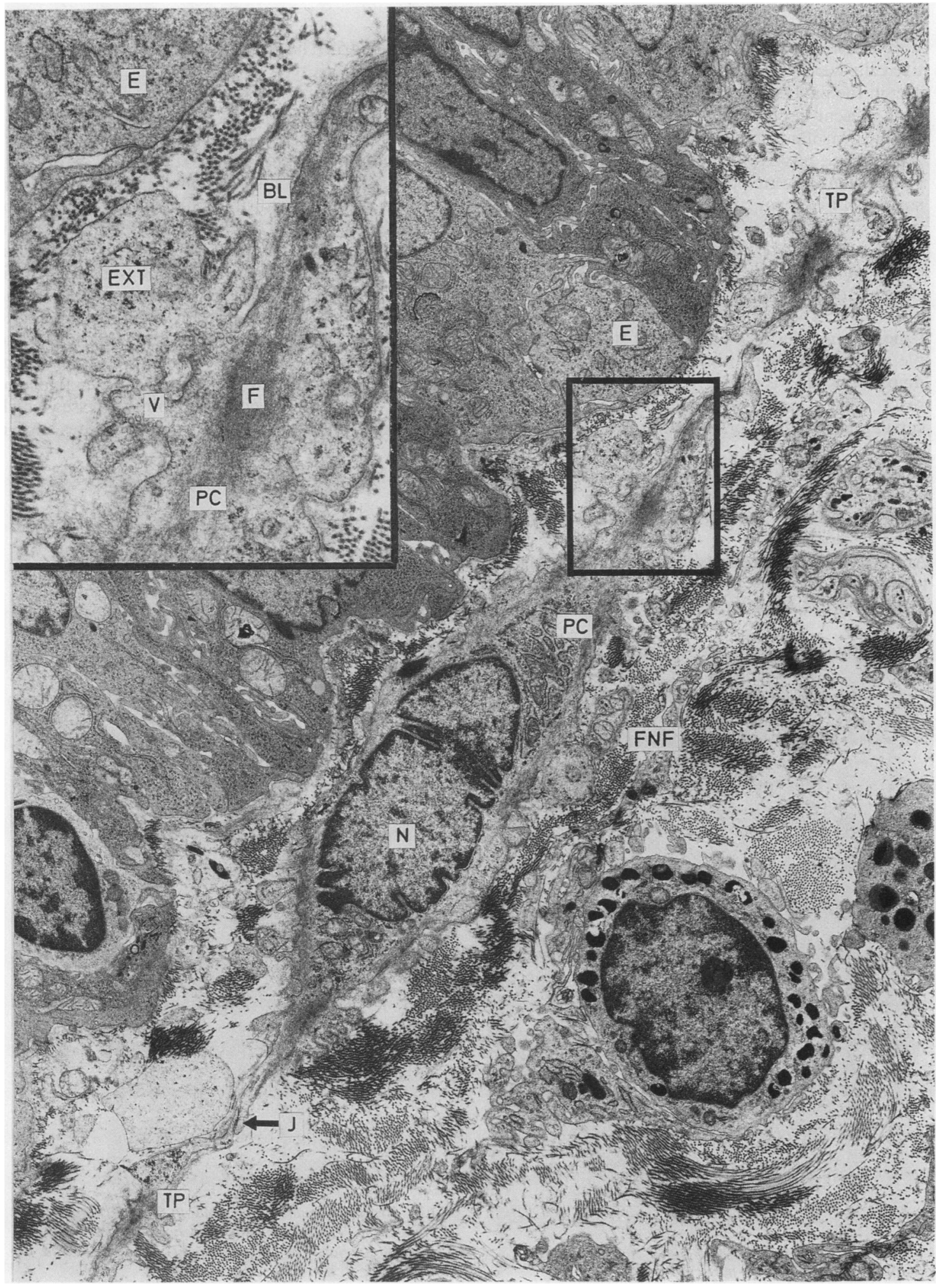

Fig 6 Pericrypt cell (PC) adjacent to crypt epithelium (E). Elongated nucleus (N) with peripheral condensation of chromatin. Aggregation of cellular organelles can be seen at nuclear poles. Long, thin processes (TP) run along basal surface of crypt. Simple junction $(J)$ between two pericrypt cells. Inset: enlargement of boxed area. Pericrypt cell (PC) process showing fine filaments with focal densities $(F)$, numerous pinocytotic vesicles $(V)$, and prominent rounded extension to cytoplasm (EXT). Basal lamina is seen $(B L) . E=$ epithelial cell. 
were classified as myofibroblasts. In the breast positive cells were seen in the intralobular connective tissue; in the pancreas and salivary glands positive cells were present in connective tissue close to large ducts and also around acinar tissue. Hepatic perisinoidal cells were positive. Myometrium and prostatic smooth muscle were positive, but neither endometrial stroma nor ovarian stroma were stained. In the testis positive cells were seen around seminiferous tubules and, in the kidney, around convoluted tubules and collecting ducts. In the respiratory tract PR 2D3 stained tracheal and bronchial smooth muscle and also cells in the alveolar septa. The erector pili (smooth) muscles of the skin were stained. In a sample of umbilical cord taken at full term, the stellate myofibroblasts were positive (fig 4). PR 2D3 did not react with skeletal or cardiac muscle, epithelium, cartilage, fibroblasts or nerve fibres.

IMMUNOCYTOCHEMICAL REACTION OF COLONIC PERICRYPT CELLS WITH ANTIBODIES TO

DESMIN, MYOSIN, ACTIN BINDING PROTEIN AND TYPE IV COLLAGEN

Antibodies to desmin, myosin, and actin binding protein stained the pericrypt cells in addition to smooth muscle of the muscularis mucosae and muscularis

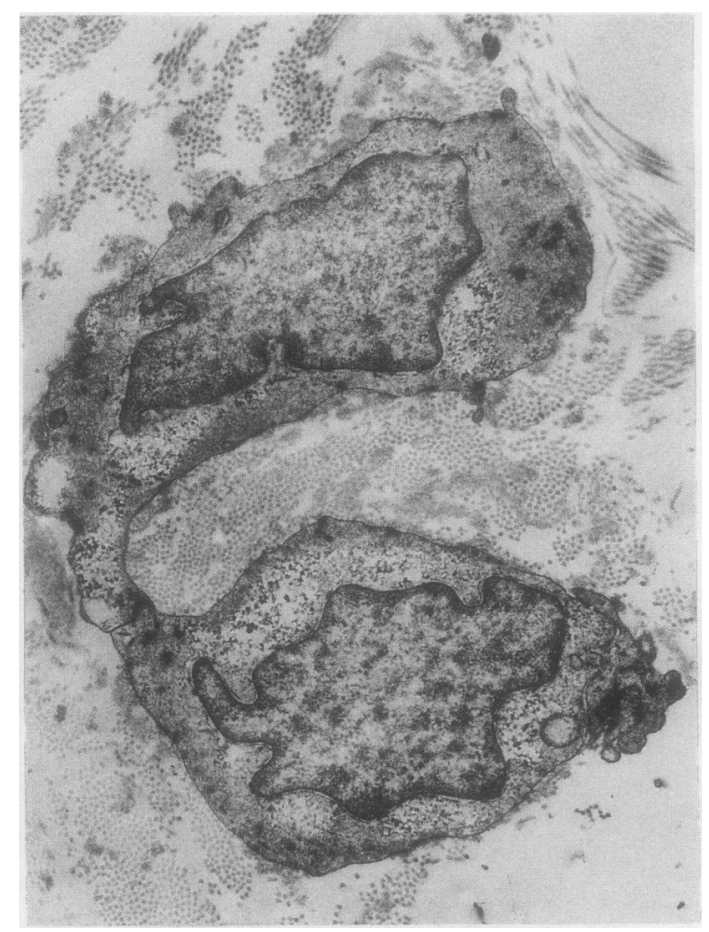

Fig 7 Umbilical cord myofibroblasts showing many features in common with pericrypt cells. propria (fig 5). The pericrypt cells were also stained by the antibody to type IV collagen.

ULTRASTRUCTURAL EXAMINATION OF THE PERICRYPT CELLS: COMPARISON WITH THE MYOFIBROBLASTS OF THE UMBILICAL CORD The pericrypt cells showed many ultrastructural features in common with the umbilical cord myofibroblasts (figs 6 and 7). Both types of cell contained an elongated nucleus with rounded ends and peripheral condensation of chromatin. Nucleoli were not prominent. The cellular organelles were aggregated at the nuclear poles and comprised small to moderate amounts of rough endoplasmic reticulum and some mitochondria. Some cells contained a small Golgi apparatus, although this was rare. The remainder of the cell consisted of very long, thin processes which extended away from the nucleus on both sides and, in

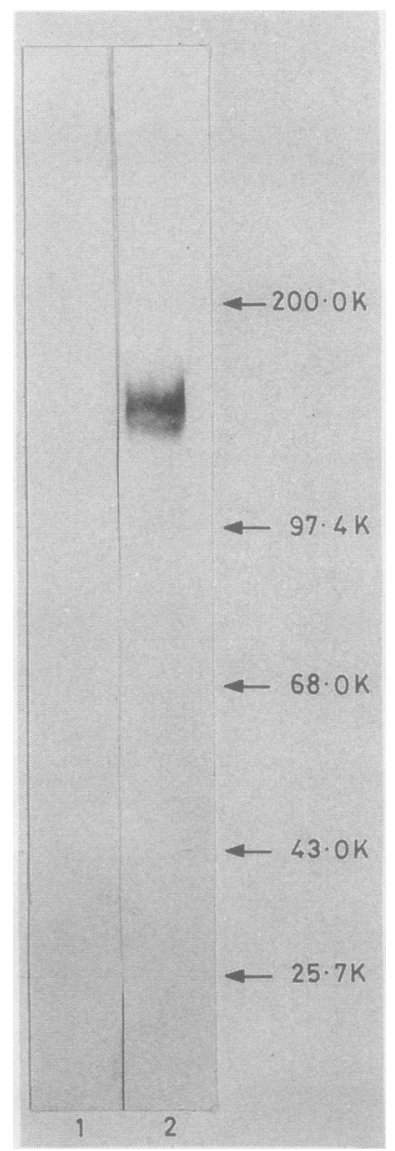

Fig 8 Protein immunoblot of smooth muscle lysate with antibody PR 2D3 showing two closely associated bands of about $140 \mathrm{~K}$ relative molecular weight. Lane 1 , reduced sample; lane 2, non-reduced sample. 
the case of the pericrypt cells, ran along the basal surface of the adjacent crypts. Fine filaments with dense focal points along them were arranged in the long axis of the cells. There were subplasmalemmal densities along the cell membrane and many cells had abundant pinocytotic vesicles. There were prominent rounded extensions of the cell cytoplasm on both sides of the cells. Many cells were surrounded by a distinct basal lamina, and simple cell junctions were present. In some areas nerve fibres were closely apposed to the pericrypt cells.

Colon tissues were sectioned in both longitudinal and transverse planes. This showed that, in addition to the cells orientated in the long axis of the crypts, other cells encircled them. The pericrypt cells thus seem to form a basket like network around the crypts.

\section{REACTION OF PR 2D3 WITH COLORECTAL}

\section{TUMOURS}

Preliminary analysis of PR 2D3 reactivity in tumours showed that adenomas and well differentiated carcinomas retained the close relation between the pericryptal and epithelial cells seen in the normal colon. This was increasingly disrupted in more poorly differentiated carcinomas, with an eventual absence of the pericrypt cells. Some carcinomas, however, were associated with high concentrations of these myofibroblasts in a disorganised fashion, consistent with a strong induction.

\section{CHARACTERISATION OF THE ANTIGEN}

DETECTED BY PR 2D3

Protein immunoblotting showed that PR 2D3 reacted with two closely associated bands of about $140 \mathrm{~K}$ relative molecular weight. Antigenic activity was lost after boiling, suggesting that the antigen is probably a protein (fig 8).

\section{Discussion}

The pericrypt cells of the large intestine were described first by Deane in $1964^{6}$ and then by Donnellan. ${ }^{7}$ Kaye et $\mathrm{l}^{3}$ provided ultrastructural evidence that these cells were fibroblasts, which, together with surrounding collagen and mucopolysaccharide ground substance, form the pericryptal fibroblast sheath. The ultrastructural features and kinetic characteristics of these cells have been described in several species and tissues, ${ }^{38-12}$ although their function has remained unclear. We have presented ultrastructural and immunocytochemical evidence that the pericrypt cells are indistinguishable from smooth muscle cells or myofibroblasts and suggest, therefore, that these cells belong to the smooth muscle myofibrolast lineage.

The term "myofibroblast" was first used by Majno in 1971 to describe a cell type with the morphological features of both smooth muscle and fibroblasts. ${ }^{13}$ Such cells have been described in many normal sites, as well as in granulation and repair tissues and in various tumours. ${ }^{14}$ Myofibroblasts have, however, not previously been described in the normal mucosa of the colorectum. Balázs and Kovács ${ }^{15}$ found them in the transitional mucosa adjacent to a colonic carcinoma, and in a recent study of four cases of collagenous colitis the pericrypt cells were shown to have the ultrastructural characteristics of myofibroblasts, ${ }^{16}$ although in that study the pericrypt cells of the normal control patients were described as fibroblasts.

Although morphologically the pericrypt cells resemble fibroblasts, and ultrastructurally, like fibroblasts, contain moderate amounts of rough endoplasmic reticulum, we found that they also share many features with smooth muscle; they contain microfilaments with dense bodies and subplasmalemmal densities, and have intercellular junctions. The pericrypt cells also resembled smooth muscle in their immunocytochemical reactions. They were stained by the monoclonal antibody PR 2D3 and contained the intermediate filament desmin, as well as myosin and the actin binding protein filamin. Filamin has recently been shown to be localised in the intermediate filament domain and the cell border at the inner surface of the plaques associated with plasmalemma of smooth muscle cells. ${ }^{17}$

Previous studies of the pericrypt cells have considered the question of cell proliferation. Uptake of tritiated thymidine by these cells has been reported, ${ }^{8-12}$ but there have been conflicting reports as to whether the pericrypt cells migrate en bloc with the epithelial cells. Pascal et al $^{8}$ and Parker et al, ${ }^{9}$ on the basis of their labelling studies in rabbits, proposed that the mesenchymal cells migrated in synchrony with the epithelial cells. These studies were not, however, confirmed by the work of Maskens et al in rats ${ }^{12}$ nor by that of Neal and Potten in mice. ${ }^{11}$ Maskens viewed the pericrypt sheath as a stable structure on which the epithelial elements migrate while undergoing differentiation.

The function of the pericrypt cells is still not clear. It has been postulated that they participate in epithelial and mesenchymal interactions with the adjacent epithelial cells, and that they may have a role in structural support and in the control of epithelial cell differentiation. ${ }^{818}$ We have shown that the pericrypt cells have characteristics of smooth muscle and myofibroblasts, which suggests that their functions may include both contraction and secretion, because smooth muscle cells and myofibroblasts are contractile. ${ }^{13}$ Contraction of the cells could aid the release of mucus from the crypt lumen, and as con- 
tractile cells they could also be targets for the products for the products of underlying endocrine cells ${ }^{19}$ and nerve cells within the lamina propria. Myofibroblasts in other sites synthesise and secrete collagen precursors, elastic fibres, proteoglycans and an external lamina, ${ }^{1420}$ and those in the umbilical cord secrete the extracellular matrix known as Wharton's jelly. ${ }^{1}$ The pericrypt cells in the colon and rectum could, therefore, be responsible for the production of extracellular matrix material. This may influence the growth and differentiation of epithelial cells of the adjacent crypts in the same way that changes in the extracellular matrix have been shown to affect the secretion of milk proteins in normal breast epithelium. ${ }^{21}$

The close association of presumed contractile and secretory pericryptal cells with the crypt epithelium emphasises the importance of specific cellular interactions in the formation of such structures. The disruption of this organised relation may be an important step in carcinogenesis. The occurrence of a myofibroblast reaction in some tumours, however, suggests that tumour epithelial cells and myofibroblasts are sometimes mutually dependent; one or more epithelial factors may recruit and stimulate myofibroblast proliferation, while myofibroblasts release epithelial growth factors. The antigen detected in the membrane by antibody PR 2D3 may be either a growth factor or a differentiation factor receptor, or it may be concerned with specific intercellular adhesion between smooth muscle cells and myofibroblasts. Further characterisation of the molecule recognised by PR 2D3, and its role in smooth muscle and myofibroblast functions - especially their interactions with epithelial cells in colonic crypts - are now being investigated.

We thank Dr Sharon Richman for invaluable discussion and for typing the manuscript.

\section{References}

I Parry EW. Some electron microscope observations on the mesenchymal structures of full-term umbilical cord. $J$ Anat 1970;107:505.

2 Kapanci Y, Assimacopoulos A, Irle C, Zwahlen A, Gabbiani G. Contractile interstitial cells in pulmonary alveolar septa: a possible regulator of ventilation/perfusion ratio? $\mathrm{J}$ Cell Biol 1974;60:375.

3 Kaye GI, Lane N, Pascal RR. Colonic pericryptal fibroblast sheath: replication, migration and cytodifferentiation of a mesenchymal cell system in adult tissue. II. Fine structural aspects of normal rabbit and human colon. Gastroenterology 1968;54:852-65.

4 Ouchterlony $O$, Nilsson LA. Immunodiffusion and immunoelectrophoresis. In: Weir DM, ed. Handbook of experimental immunology. Oxford: Blackwell Scientific Publications, 1978:19.

5 Towbin H, Staehelin T, Gordon J. Electrophoretic transfer of proteins from polyacrylamide bells to nitrocellulose sheets: procedure and some applications. Proc Natl Acad Sci USA 1979;76:4350-4.

6 Deane HW. Some electron microscopic observations on the lamina propria of the gut, with comments on the close association of macrophages plasma cells and eosinophils. Anat Rec 1964;149:453-73.

7 Donnellan WL. The structure of the colonic mucosa. The epithelium and subepithelial reticulohistiocytic complex. Gastroenterology 1965;49:496-514.

8 Pascal RR, Kaye GI, Lane N. Colonc pericryptal fibroblast sheath: replication, migration and cytodifferentiation of a mesenchymal cell system in adult tissue. I. Autoradiographic studies of normal rabbit colon. Gastroenterology 1968;54:835-51.

9 Parker FG, Barnes EN, Kaye GI. The pericryptal fibroblast sheath. IV. Replication, migration and differentiation of the subepithelial fibroblasts of the crypt and villus of the rabbit jejunum. Gastroenterology 1974;67:607-21.

10 Marsh MN, Trier JS. Morphology and cell proliferation of the subepithelial fibroblasts in adult mouse jejunum. I. Structura features. Gastroenterology 1974;67:622-35.

11 Neal JV, Potten CS. Description and basic cell kinetics of the murine pericryptal fibroblast sheath. Gut 1981;22:19-24.

12 Maskens AP, Rahier JR, Meersseman FP, Dujardin-Loits R-M, Haot JG. Cell proliferation of pericryptal fibroblasts in the rat colon mucosa. Gut 1979;20:775-9.

13 Majno G, Gabbiani G, Hirschel BJ, Ryan GB, Statkov PR. Concentration of granulation tissue in vitro: similarity to smooth muscle. Science 1971;173:548-50.

14 Ghadially FN. Ultrastructural pathology of the cell and matrix. 2nd ed. London: Butterworths, 1982:658.

15 Balázs M, Kovács A. The "transitional" mucosa adjacent to large bowel carcinoma-electron microscopic features and myofibroblast reaction. Histopathology 1982;6:617-29.

16 Hwang WS, Kelly JK, Shaffer EA, Hershfield NB. Collagenous colitis: a disease of the pericryptal fibroblast sheath? $J$ Patho 1986;149:33-40.

17 Small JV, Fürst DO, De Mey J. Localisation of filamin in smooth muscle. J Cell Biol 1986;102:210-20.

18 Mathan M, Hermos JA, Trier JS. Structural features of the epithelio-mesenchymal interface of rat duodenal mucosa dur ing development. J Cell Biol 1972;52:577-88.

19 Polack JM, Bloom SR. Localisation of regulatory peptides in the gut. Br Med Bull 1982;38:303-8.

20 Wissler RW. The arterial medial cell, smooth muscle or multifunctional mesenchyme? Circulation 1967;36:1.

21 Lee EY-H, Parry G, Bissel MJ. Modulation of secreted protein of mouse mammary epithelial cells by the collagenous substrata. J Cell Biol 1984;98:146-55.

Requests for reprints to: Dr PI Richman, Clinical Research Fellow, Director's Laboratory, Imperial Cancer Research Fund, London WC2A 3PX, England. 